\title{
Research on factors that influence the course of canine parvovirosis
}

\author{
ALICJA WÓJCIK, STANISŁAW WINIARCZYK, ŁUKASZ ADASZEK \\ Department of Epizootiology and Clinic of Infectious Diseases, Faculty of Veterinary Medicine, \\ University of Life Sciences in Lublin, Głęboka 30, 20-612 Lublin, Poland
}

Wójcik A., Winiarczyk S., Adaszek Ł.

\section{Research on factors that influence the course of canine parvovirus}

\section{Summary}

The aim of this study was to demonstrate correlations of the course of canine parvovirus infection and the rate of cytopathic effect (CPE) on CPV-infected CCC clone 81 cell lines with the age of the affected animals, their history of CPV vaccination and the strain of the virus causing the infection. Forty-five dogs with parvovirus confirmed by rapid diagnostic tests and PCR were studied. Faecal samples from diseased animals were used to infect cell lines, which were then observed for a cytopathic effect. The statistical analysis of the results obtained demonstrated a positive correlation between the recovery rate of the diseased animals and the fact that they had been vaccinated against CPV. In dogs that had been vaccinated against CPV, the mean duration of the disease was 6 days, whereas in the group of unvaccinated dogs it was 7 days. The course of the disease also depended on the virus strain. It was shown that dogs from which the CPV-2a strain was isolated took longer to recover, had more severe clinical signs and higher mortality. The cytopathic effect appeared in all infected cultures, while the rate at which changes occurred was independent of the virus strain with which the cells were infected, the age of the dogs from which CPV was isolated and their CPV vaccination history. The study results obtained may serve as valuable prognostic guidelines as to the duration of and recovery from canine parvovirus infection.

Keywords: CPV, dogs, cell culture

Canine parvovirosis is a viral disease, the etiological agent of which is CPV-2 (canine parvovirus type 2) belonging to the family Parvoviridae, subfamily Parvovirinae, genus Protoparvovirus (18).

The first cases of CPV-2 infection in dogs were reported in 1978 in the United States. The virus, due to its high contagiousness and low sensitivity to environmental conditions, spread quickly throughout the world. Over the years, CPV has evolved, and currently three strains are distinguished: CPV-2a, CPV-2b and CPV-2c (2), which are widely accepted to have almost completely replaced CPV-2 (11).

The virus enters the dog's body by ingestion, through contact with the vomit or faeces of sick animals. Due to the high resistance of parvovirus strains to environmental conditions, infection can occur not only through direct contact, but also indirectly, through contaminated items, such as bowls or toys.

Canine parvovirosis is a systemic disease, and the virus has a special affinity to rapidly dividing cells. After penetrating the body, it initially replicates in the lymphoid tissue of the throat, from where it spreads to the intestinal crypts. The typical signs of parvovirus include apathy, lack of appetite, vomiting and bloody diarrhoea $(3,7)$. The disease often leads to the death of infected animals (13).

Not all dogs are equally susceptible to infection. Factors predisposing to the development of infection are age (with peak susceptibility between 6 weeks and 6 months of age), stress (caused by poor hygiene conditions, high population density or, for example, a change in the place where the dog lives and its separation from its mother) and, most of all, the lack of or incorrectly conducted vaccinations against this disease, which are considered to be the most effective form of its prevention (9). The course of parvovirosis is also determined by the virulence of the CPV strain responsible for the infection.

The aim of this study was to demonstrate correlations of the course of canine parvovirosus infection and the rate of cytopathic effect (CPE) on CPV-infected CCC clone 81 cell lines with the age of the affected animals, their history of anti-CPV vaccination and the strain of the virus causing the infection. 


\section{Material and methods}

Animals used in the study. The study was performed in 2017-2019 and involved 45 dogs from eastern Poland of various breeds and both sexes (females $\mathrm{n}=19$, males $\mathrm{n}=26$ ), aged 6 weeks to 8 years, with confirmed parvovirosis. The dogs were divided into two age groups: I - up to 6 months of age and II - over 6 months of age. Fifteen dogs were presented with a completed full cycle of primary vaccinations in weeks 6,9 and 12 , while 30 animals were never vaccinated (Tab. 1). All dogs showed clinical signs of parvovirosis (apathy, fever, diarrhoea, vomiting). The disease was confirmed by speed tests based on the immunochromatography method (CPV/CCV Ag Vetexpert) and PCR performed according to a method described by Buonavoglia et al. (2001).

Faeces from the rectum were collected from all the sick animals with aseptic swabs without transport medium. The sample was then suspended in phosphate-buffered saline without $\mathrm{Ca}$ and $\mathrm{Mg}$ ions (PBS, Biomed S.A.). The mixture was vortexed and incubated for 12 hours at $4{ }^{\circ} \mathrm{C}$. It was then filtered through filters with $0.45 \mu \mathrm{m}$ and $0.22 \mu \mathrm{m}$ pores (Merck Milipore) and treated by penicillin, streptomycin and tylosin (Sigma-Aldrich).

Cell cultures. Continuous cell line $\mathrm{CCC}$ clone 81 (cat kidney fibroblast transformed by Murine Sarcoma Virus; Sigma-Aldrich) (11) was used to culture CPV. A rectal swab from a healthy dog, prepared as described above, was used as a negative control.

A mixture of growth medium (consisting of Dulbecco's Modified Eagle Medium, Fetal Bovine Serum and L-glutamine) and faecal filtrate from diseased animals was applied to the cell lines. The whole was incubated at $37^{\circ} \mathrm{C}, 4 \% \mathrm{CO}_{2}$. Cell cultures were observed daily for 10 days through an inverted microscope (Olympus CKX41 Japan) to detect changes indicative of CPE.

Molecular analysis. DNA of canine parvovirus for analysis was isolated from rectal swabs taken from diseased animals and from the supernatant of virus-infected cell cultures in which a cytopathic effect was observed using GeneAll Exgene Stool DNA mini kit. Polymerase chain reaction (PCR) was performed using a programmable thermal cycler (Biometra). The PCR reaction for CPV VP2 gene was carried out by a method described by Buonavoglia (2001) with the following primers: 555 for (positions 4003 $-4022)$ 5' $\rightarrow$ 3' CAGGAAGATATCCAGAAGGA and 555rev (positions 4585-4561) 5' $\rightarrow$ 3' GGTGCTAGTTGATATGTAATAAACA. This limited the DNA section of the VP2 gene with a length of $583 \mathrm{bp}$. The positive control was the DNA of CPV-2b (CPV-2b-Bio 12/B strain), isolated from a commercially available vaccine (Versican Plus DHPPi, Zoetis Belgium SA), and the negative control was DNA from the stool of a healthy dog. Each reaction was composed of 30 cycles with the denaturation stage at $94^{\circ} \mathrm{C}$ for $60 \mathrm{~s}$, annealing at $58^{\circ} \mathrm{C}$ for $60 \mathrm{~s}$ and elongation at $72^{\circ} \mathrm{C}$ for $90 \mathrm{~s}$. The reaction mixture $(50 \mu \mathrm{L})$ contained $100 \mu \mathrm{M}$ of each dNTP, $1.6 \mathrm{mM}$ of $\mathrm{MgCl}_{2}, 0.25 \mu \mathrm{M}$ of each primer, $2.5 \mathrm{U}$ of Taq DNA polymerase, and $5 \mu \mathrm{L}$ of DNA template. The PCR products were analysed by the electrophoresis method in a $1 \%$ agarose gel and Tris-borate-EDTA (TBE) buffer at a voltage of $10 \mathrm{~V} / \mathrm{cm}$ for 50 mins. The PCR reaction prod- ucts purified with a QIAquick PCR Purification Kit (Qiagen) were sequenced by the DNA Sequencing and Synthesis Service of the Institute of Biochemistry and Biophysics of the Polish Academy of Science in Warsaw. The sequencing results were received by email and developed using the computer software Lasergene DNA Star MegAlign (Madison, USA). The same software was used to analyse the sequence of CPV isolates and compare them with sequences available in the National Center for Biotechnology Information (NCBI) Genebank from: Germany (AY742953, AY742935), Brazil (DQ340434), South Africa (AJ007500, AJ007498), New Zealand (AY742933), China (EU145958), Turkey (EU145958, KF500499), Italy (GU362934, FJ005252, AF306447, FJ005263, FJ005195), United States (M24000, M38245, AY742955, M74849, FJ005235), France (DQ025986, DQ025992), India (AJ698134, DQ182623), South Korea (EU009206, EF599097), Japan (AB054220), Poland (Z46651), Spain (FJ005214), Belgium (FJ005247) and Greece (GQ865518, GQ865519) and with sequences VP2 of CPV strains used in commercial vaccines. Double phylogenetic analysis was performed. Phylogenetic trees were created separately for CPV VP2 amplicons obtained from vaccinated dogs and non-vaccinated dogs.

Statistical analysis. Statistical analysis was performed using the Chi-squared test. The relationships between the course of the disease and the rate at which the cytopathic effect on cell lines occurred, the strain of the virus isolated from the dogs, the history of vaccination against parvovirus and the age of the affected animals were analysed. Changes were considered statistically significant at $\mathrm{P}<0.05$. The Statistica 10.0 PL software was used for the calculations.

\section{Results and discussion}

Results of molecular analysis. Legible sequences were obtained for 45 DNA samples. The sequences of the CPV VP2 gene obtained in the study showed a mutual homology of $98.5-100 \%$.

The computer processing of CPV VP2 gene sequences from vaccinated dogs by means of the Lasergene DNA Star software made it possible to create a phylogenetic tree showing similarities between particular isolates. Differences in the primary genetic structure of each isolate allowed us to distinguish 6 monophyletic groups. One group was formed by isolates No 11, 24, 25, 27, 34 and 26. It showed the highest homology (99.5\%) with the CPV-2b strain sequence isolated in Turkey (KF500499). Another group was formed by a single strain, No 22, which showed the highest similarity of the gene sequence examined (99.4\%) to CPV-2b isolated in Korea (EU009206). A third group included isolates No 3, 17, 19, 39, which showed the highest homology (99.8-100\%) with the Japanese strain CPV-2b (AB054220). A fourth group consisted of three isolates (No 13, 14 and 18) showing the highest homology (99.3-99.4\%) with the Indian CPV-2b strain (DQ182623). The remaining group was formed by one isolate (No 15), which had the greatest similarity of the gene sequence examined $(99.8 \%)$ to the Italian CPV-2a isolate (FJ005252). The parvovirus 
Tab. 1. Summary of the study results, including a breakdown by the age group of the animals (I - up to 6 months of age and II - over 6 months of age), vaccination history, time when the cytopathic effect on cell lines occurred, CPV strain isolated, and the time of the animal's recovery

\begin{tabular}{|c|c|c|c|c|c|c|c|c|c|}
\hline Item No. & Age group & Breed & Sex & Vaccine brand & Vaccination & CPE (incubation day) & Strain & \multicolumn{2}{|c|}{ Dog recovery time } \\
\hline 1 & I & Mix & $\mathbf{F}$ & No vaccination & - & 2 & CPV-2b & 8 & days \\
\hline 2 & I & Labrador retriever & M & Nobivac $3 \mathrm{x}$ & + & 3 & CPV-2b & 7 & days \\
\hline 3 & II & German Shepherd & M & No vaccination & - & 7 & CPV-2a & 9 & days \\
\hline 4 & II & German Shepherd & M & No vaccination & - & 4 & $\mathrm{CPV}-2 \mathrm{~b}$ & 5 & days \\
\hline 5 & II & German Shepherd & M & No vaccination & - & 4 & CPV-2a & 8 & days \\
\hline 6 & I & Mix & $\mathbf{F}$ & No vaccination & - & 5 & CPV-2b & 7 & days \\
\hline 7 & II & Mix & M & No vaccination & - & 6 & CPV-2b & 7 & death \\
\hline 8 & I & Mix & M & No vaccination & - & 6 & CPV-2b & 6 & death \\
\hline 9 & I & White Swiss Shepherd & $\mathbf{F}$ & No vaccination & - & 7 & CPV-2b & 5 & days \\
\hline 10 & II & White Swiss Shepherd & $\mathbf{F}$ & Nobivac $3 x$ & + & 7 & CPV-2b & 5 & days \\
\hline 11 & I & German Shepherd & M & Biocan 2x & + & 3 & CPV-2b & 6 & days \\
\hline 12 & I & German Shepherd & M & Biocan 2x & + & 3 & CPV-2b & & death \\
\hline 13 & I & German Shepherd & $\mathbf{M}$ & Biocan 2x & + & 5 & CPV-2a & & death \\
\hline 14 & II & Maltese & M & Vanguard, Canigen & + & 3 & CPV-2b & & death \\
\hline 15 & I & Mix & M & Biocan & + & 7 & CPV-2b & 7 & days \\
\hline 16 & I & Mix & $\mathbf{F}$ & No vaccination & - & 5 & CPV-2b & & death \\
\hline 17 & I & Mix & $\mathbf{F}$ & No vaccination & - & 0 & CPV-2b & 8 & days \\
\hline 18 & I & Mix & $\mathbf{F}$ & Nobivac & + & 5 & CPV-2b & 7 & days \\
\hline 19 & II & Mix & M & No vaccination & - & 7 & CPV-2b & 8 & days \\
\hline 20 & I & Mix & M & Nobivac & + & 3 & CPV-2b & 8 & days \\
\hline 21 & I & Mix & M & Nobivac & + & 6 & CPV-2b & & death \\
\hline 22 & I & Mix & $\mathbf{F}$ & Nobivac & + & 7 & CPV-2b & 8 & days \\
\hline 23 & I & Mix & $\mathbf{F}$ & Nobivac & + & 6 & CPV-2b & 8 & days \\
\hline 24 & I & Maltese & M & No vaccination & - & 7 & CPV-2b & 7 & death \\
\hline 25 & II & Maltese & $\mathbf{F}$ & No vaccination & - & 6 & CPV-2b & 6 & days \\
\hline 26 & II & Czechoslovakian Wolfdog & M & No vaccination & - & 7 & CPV-2b & 6 & days \\
\hline 27 & I & Mix & $\mathbf{F}$ & Biocan & + & 5 & CPV-2b & & death \\
\hline 28 & I & Rottweiler & $\mathbf{F}$ & Biocan & + & 7 & CPV-2b & 5 & days \\
\hline 29 & I & Welsh Terrier & M & No vaccination & - & 4 & CPV-2b & 7 & days \\
\hline 30 & II & Mix & M & No vaccination & - & 7 & CPV-2b & 6 & days \\
\hline 31 & II & Mix & M & No vaccination & - & 6 & CPV-2b & & death \\
\hline 32 & II & Mix & $\mathbf{F}$ & No vaccination & - & 4 & CPV-2b & 8 & days \\
\hline 33 & I & Mix & $\mathbf{F}$ & Nobivac & + & 4 & CPV-2b & & death \\
\hline 34 & I & Mix & M & Nobivac & + & 4 & CPV-2b & 4 & days \\
\hline 35 & I & German Shepherd & M & No vaccination & - & 3 & CPV-2b & 6 & days \\
\hline 36 & I & Mix & M & No vaccination & - & 7 & CPV-2b & & death \\
\hline 37 & I & White Swiss Shepherd & $F$ & No vaccination & - & 3 & CPV-2b & 7 & days \\
\hline 38 & I & Mix & M & No vaccination & - & 3 & CPV-2a & & death \\
\hline 39 & I & Mix & $\mathbf{F}$ & No vaccination & - & 7 & CPV-2b & 6 & days \\
\hline 40 & I & Mix & $F$ & No vaccination & - & 3 & CPV-2a & & death \\
\hline 41 & I & Mix & $\mathbf{F}$ & No vaccination & - & 3 & CPV-2a & 7 & days \\
\hline 42 & II & Mix & M & No vaccination & - & 3 & CPV-2a & 9 & days \\
\hline 43 & II & Mix & $F$ & No vaccination & - & 5 & CPV-2b & & death \\
\hline 44 & I & Mix & M & No vaccination & - & 3 & CPV-2a & 6 & days \\
\hline 45 & I & Mix & M & Nobivac & + & 3 & CPV-2b & & death \\
\hline
\end{tabular}




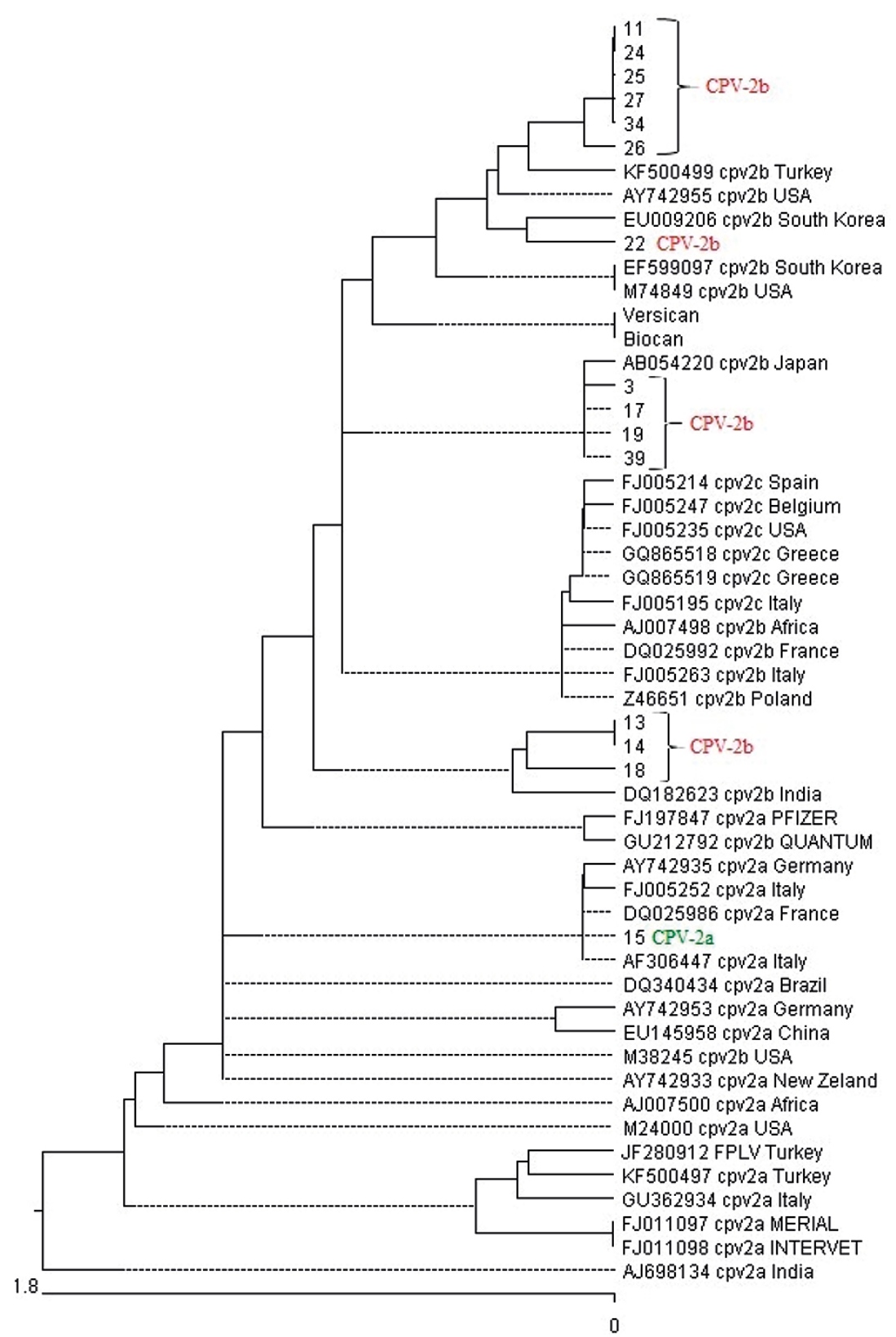

Fig. 1. Phylogenetic tree of CPV strains isolated from dogs which had received preventive vaccination

strains isolated from vaccinated dogs in which the disease developed were classified in most cases $(14 / 15)$ as CPV-2b, and in one case as CPV-2a (Fig. 1).

Phylogenetic analysis of the VP2 CPV sequences obtained from the group of nonvaccinated dogs (Fig. 2) revealed 5 monophyletic groups. One group consisted of 11 isolates (No 2, 10, 28, 33, 35, 36, 37, 38, $40,42,53)$ showing the highest similarity (99.8-100\%) to the CPV-2b virus sequence isolated in Japan (AB054220). Another group was formed by a single strain (No 46) most homologous $(99.8 \%)$ with CPV$2 \mathrm{~b}$ isolated in Italy (FJ005263). A third group was formed by isolates 23, 29 and 41 , which showed the highest homology

from dogs which had
(99.8\%) with the CPV-2b virus isolated in South Korea (EU009206). A fourth group was formed by 8 isolates (No 5, 7, 8, 9, $20,21,30$ and 51) showing the highest similarity of the VP2 gene sequence analysed (99.4-99.8\%) to sequence DQ182623 from India. The remaining group consisted of isolates No 4, 6, 44, 48, 49, 50 and 52,

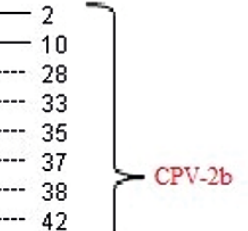

$$
\begin{aligned}
& \text { AB054220 cpv2b Japan } \\
& \text {--- } 53 \\
& -40 \\
& \begin{array}{r}
40 \\
-\quad 36
\end{array}
\end{aligned}
$$

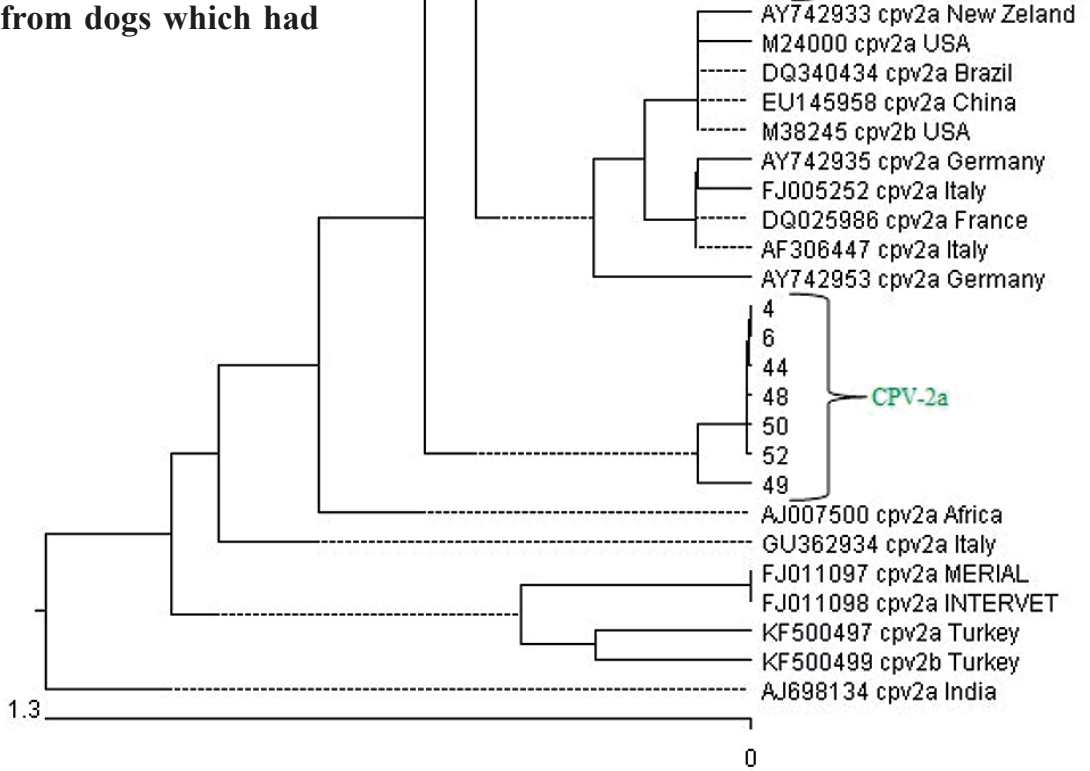

Fig. 2. Phylogenetic tree of CPV strains isolated from dogs which had not received preventive vaccination 
which were most homologous (99.6-99.8\%) with the CPV-2a strains from New Zealand (AY742933), China (EU145958) and Brazil (DQ340434). As in the group of vaccinated dogs, the main CPV strain isolated from non-vaccinated dogs with signs of parvovirosis was CPV-2b (23/30). In the remaining seven cases, the etiological factor of the infectious agent of the disease was CPV-2a.

A total of 8 dogs were found to have developed the CPV-2a strain, whereas 37 developed the CPV-2b strain.

Results of cytopathic effect observation. The cytopathic effect occurred in all 45 cell cultures infected with the test material. The changes were characterised by the rounding of the cells, syncytia formation and partial lysis of the monolayer until complete separation from the growth surface of the bottle (Fig. 3). Depending on the viral inocula used, the cytopathic effect occurred at different times, between 2 and 7 days after the infection of cell lines.

Results of statistical analysis. The statistical analysis of the results obtained did not show significant differences in the severity of clinical signs or the recovery time between animals from age groups I and II $(\mathrm{P}=0.58)$. However, a positive correlation was found between the speed of recovery and the fact that the animal had been vaccinated $(\mathrm{P}=0.001)$. In dogs that had been vaccinated against $\mathrm{CPV}$, the mean duration of the disease was 6 days, whereas in the group of unvaccinated dogs it was 7 days. The course of the disease also depended on the virus strain $(\mathrm{P}=0.006)$. It was shown that dogs from which the CPV-2a strain was isolated took longer to recover, had more severe clinical signs and higher mortality rates. Among dogs from which the CPV-2a strain was isolated, the mortality rate was $37.5 \%$ ( 3 out of 8 dogs), whereas among the dogs from which the CPV-2b strain was isolated the mortality rate was $31.58 \%(12 / 37)$.

The cytopathic effect appeared in all infected cultures, and the rate at which changes occurred was independent of the virus strain with which the cells were infected (CPV-2a or CPV-2b) $(\mathrm{P}=0.41)$, the age of the dogs from which CPV was isolated $(\mathrm{P}=0.43)$ or the dogs' CPV vaccination history $(\mathrm{P}=0.63)$. Table 1 presents data on the rate at which the cytopathic effect occurred after the infection of cell lines with viral inocula obtained from the dogs used in the study.

Canine parvovirosis is a viral disease that occurs worldwide (11). The best method of prevention is a widespread vaccination of the canine population. Its purpose is to generate or strengthen specific immune mechanisms that, together with non-specific response mechanisms, protect dogs against the disease. Animal immunity is determined by in vitro tests, with which a satisfactory result can be obtained in a manner that is a minimally invasive for the animal tested. The immune response against the different variants of canine parvovirus is cross-reactive, so that vaccines currently avail-

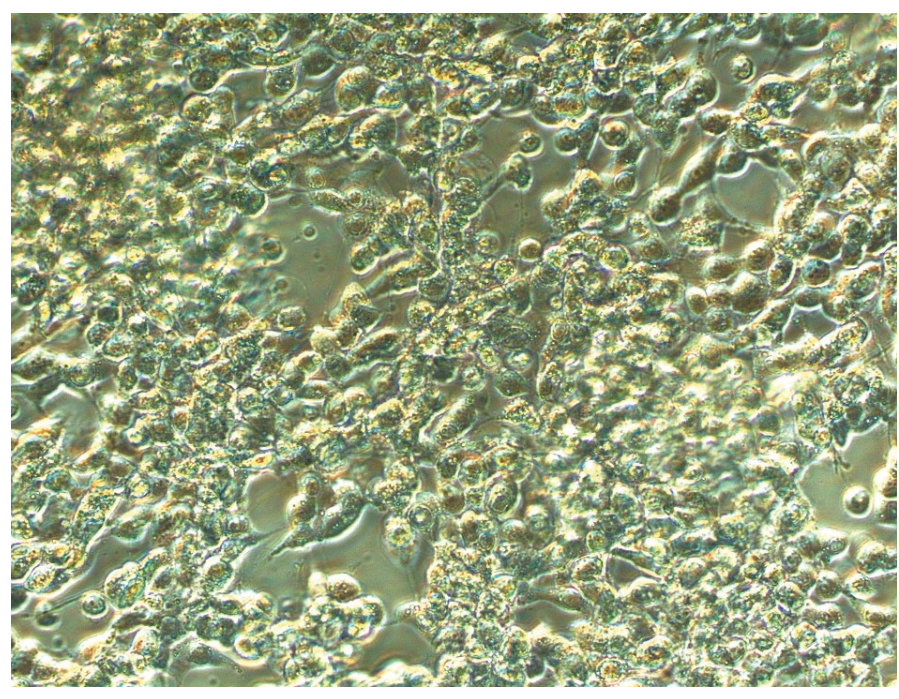

Fig. 3. Cytopathic effect on cell lines CCC clone 81

able on the market are regarded as effective protection against the disease caused by each of the existing virus variants (CPV-2, CPV-2a, CPV-2b and CPV-2c) (19). No vaccination offers complete protection from the disease, but thanks to cross-resistance between different strains of canine parvovirus, vaccinated animals should be affected less severely. This is confirmed by our results, which showed statistically significant differences between the recovery rates of the vaccinated and unvaccinated groups of dogs. The animals that were infected with the CPV-2a strain took longer to recover than the dogs from which the CPV-2b strain was isolated. The mortality rate of the dogs from which the CPV-2a strain was isolated was also higher than that of the other group, although not different from the mortality rates reported by other authors, ranging from $27.8 \%$ to $93.5 \%$ (17).

The dogs between 6 weeks and 6 months of age are considered to be the most susceptible age group $(7,9)$, and the most severe course of the disease usually occurs in puppies up to 3 months of age $(1,6)$. Despite this, no statistically significant differences in recovery time were demonstrated between animals up to 6 months of age and older animals. This may be due to the intensive treatment of all sick animals as well as the improvement in treatment and early diagnosis since the time when the studies cited above were conducted.

Cytopathic effect is a set of changes that occur in cell cultures under the influence of a specific factor. In our study, the agent was canine parvovirus, and the cytopathic effect was characterised by the rounding of cells, syncytia formation and partial lysis of the monolayer until complete separation from the growth surface of the bottle. The effect occurred, at the earliest, 2 days after the infection of the culture. CPV was cultured on CCC clone 81 lines, on which canine parvovirus multiplies easily and cytopathic changes are well expressed (10). It seems that on other lines, such as CRFK (15), FCH (16) and A-72 (5), the cytopathic effect is more difficult to observe. Our study showed 
no statistically significant differences in the rate at which the cytopathic effect occurred depending on the virus strain, the age of the dogs from which CPV was isolated or their vaccination history. So far, this type of analysis has not been conducted. It might seem that the cytopathic effect should occur faster and be more intense in cell cultures infected with more virulent CPV strains. Our observations, however, did not show any differences in the severity of CPE in cultures infected with CPV-2a and CPV-2b. This may be due to the fact that isolation of parvovirus is difficult, and the cytopathic effect may be poorly expressed in cell culture. CPV virions are often captured by specific antibodies in the intestinal lumen, which prevents their adsorption to intestinal epithelial cells. Consequently, multiplication of faeces-derived parvovirus on cell lines may be hindered $(4,14)$.

Our research revealed that the main strains of parvoviruses currently occurring in dogs in Poland are CPV-2a and CPV-2b. This situation has been changing dynamically over the years. In 1995, Mizak and Plucienniczak examined CPV strains isolated between 1982 and 1993 and demonstrated that the dominant strain in the dog population in Poland at that time was CPV-2a. On the other hand, Majer-Dziedzic et al. showed that CPV-2c was dominant in 2011, whereas Kowalczyk et al. found that CPV 2a was the most prevalent strain in 2019. Our research, for which material was collected between 2017 and 2019, showed that the dominant variant in the dog population in eastern Poland was CPV-2b. The results of all above studies show dynamic changes in dominant parvovirus strains $(8,10,12)$.

It seems that the virus strain causing parvovirosis in a given individual and the history of vaccination against canine parvovirosis can be valuable prognostic markers for the duration of the disease and the probability of death in its course. According to our observations, the age of the animal has no significant effect on the rate of recovery, whereas the fact that the animal has been vaccinated makes recovery faster. On the other hand, the observation of the rate at which the cytopathic effect occurs does not provide any relevant information for the clinical picture of canine parvovirosis.

\section{References}

1. Appel M. J., Scott F. W., Carmichael L. E.: Isolation and immunisation studies of a canine parvo-like virus from dogs with heamorrhagic enteritis. Vet. Rec. 1979, 105, 156-159.

2.Buonavoglia C., Martella V., Pratelli A., Tempesta M., Cavalli A., Buonavoglia D., Bozzo G., Elia G., Decaro N., Carmichael L.: Evidence for evolution of canine parvovirus type 2 in Italy. J. Gen. Virol. 2001, 82, 3021-3025 .

3. Castro T. X., Miranda S. C., Labarthe N. V., Silva L. E., Cubel Garcia R. C. N.: Clinical and epidemiological aspects of canine parvovirus (CPV) enteritis in the State of Rio de Janeiro: 1995-2004. Arq. Bras. Med. Veterinária E Zootec. 2007, 59, 333-339.

4. Decaro N., Desario C., Campolo M., Elia G., Martella V., Ricci D., Lorusso E., Buonavoglia $C$.: Clinical and virological findings in pups naturally infected by canine parvovirus type 2 Glu-426 mutant. J. Vet. Diagn. Invest. 2005, 17 , $133-138$.
5. Flávia V. Vieira, Daniel J. Hoffmann, Carolina U. F. Fabri, Katia D. S. Bresciani, Roberto Gameiro, Eduardo F. Flores, Tereza C. Cardoso: Circulation of canine parvovirus among dogs living in human-wildlife interface in the Atlantic forest biome, Brazil. Heliyon. 2017, 3, e00491.

6. Jacob R. M., Weiser M. G., Hall R. L., Kowalski J. J.: Clinic pathogenic features of canine parvoviral enteritis. J. Am. Vet. Med. Assoc. 1980, 16, 809-813.

7. Kalli I., Leontides L. S., Mylonakis M. E., Adamama-Moraitou K., Rallis T., Koutinas A. F.: Factors affecting the occurrence, duration of hospitalization and final outcome in canine parvovirus infection. Res. Vet. Sci. 2010, 89, 174-178.

8.Kowalczyk M., Majer-Dziedzic B., Kostro K., Szabelak A., Ziętek J., Gryzinska M., JakubczakA.: Diagnostics and genotyping of Canine parvovirus type 2 (CPV-2) from disease cases in south-eastern Poland. Acta Vet. 2019, 69, 32-46.

9. Larson L. J., Schultz R. D.: Do two current canine parvovirus type 2 and $2 b$ vaccines provide protection against the new type $2 \mathrm{c}$ variant? Vet. Ther. 2008, 9, 94-101.

10. Majer-Dziedzic B., Jakubczak A., Ziętek J.: Phylogenetic analysis of Canine parvovirus CPV-2 strains and its variants isolated in Poland. Pol. J. Vet. Sci. 2011, 14, 379-384.

11. Miranda C., Thompson G.: Canine parvovirus: the worldwide occurrence of antigenic variants. J. Gen. Virol. 2016, 97, 2043-2057.

12. Mizak B., Plucienniczak A.: Antigenic typing Polish isolates of canine parvovirus. Bulletin of the Veterinary Institute in Pulawy 1995, 39, 71-76.

13. Nandi S., Kumar M.: Canine parvovirus: current perspective. Indian J. Virol. 2010, 21, 31-44.

14. Ohshima T., Mochizuki M., Kawakami K., Kishi M., Tohya Y., Mochizuki M. Chronological Analysis of Canine Parvovirus Type 2 Isolates in Japan. J. Vet. Med. Sci. 2008, 70, 769-775.

15. Parthiban S., Mukhopadhyay H. K., Panneer D., Antony P. X., Pillai R. M.: Isolation and Typing of Canine Parvovirus in CRFK Cell Line in Puducherry, South India. Indian J. Microbiol. 2011, 51, 456-460.

16. Puentes R., Eliopulos N., Pérez R., Franco G., Sosa K., Bianchi P., Furtado A., Hubner $S$. O., Esteves $P$. A.: Isolation and characterization of canine parvovirus type 2C (CPV-2C) from symptomatic puppies. Braz. J. Microbiol. 2012, 43, 1005-1009.

17. Qi S., Zhao J., Guo D., Sun D.: A Mini-Review on the Epidemiology of Canine Parvovirus in China. Front Vet. Sci. 2020, 7, 5.

18. Ros C., Bayat N., Wolfisberg R., Almendral J. M.: Protoparvovirus Cell Entry. Viruses 2017, 9, 313.

19. Wilson S., Illambas J., Siedek E., Stirling C., Thomas A., Plevová E., Sture G. Salt J.: Vaccination of dogs with canine parvovirus type $2 \mathrm{~b}(\mathrm{CPV}-2 \mathrm{~b})$ induces neutralising antibody responses to CPV-2a and CPV-2c. Vaccine 2014, 32 5420-5424.

Corresponding author: Alicja Wójcik, DVM, Department of Epizootiology and the Clinic of Infectious Diseases, Faculty of Veterinary Medicine, University of Life Sciences in Lublin, Głęboka 30, 20-612 Lublin, Poland; e-mail: lis.alicja@gmail.com 